\title{
Transplante de córnea e o conhecimento do procedimento pelos pacientes
}

\author{
Corneal transplantand what the patientsknow about it
}

\author{
Gerson López Moreno ${ }^{1}$ \\ Luciene Barbosa de Souza ${ }^{2}$ \\ Denise de Freitas ${ }^{3}$ \\ Elcio Hideo Sato ${ }^{4}$ \\ Luis Antonio Vieira ${ }^{4}$
}

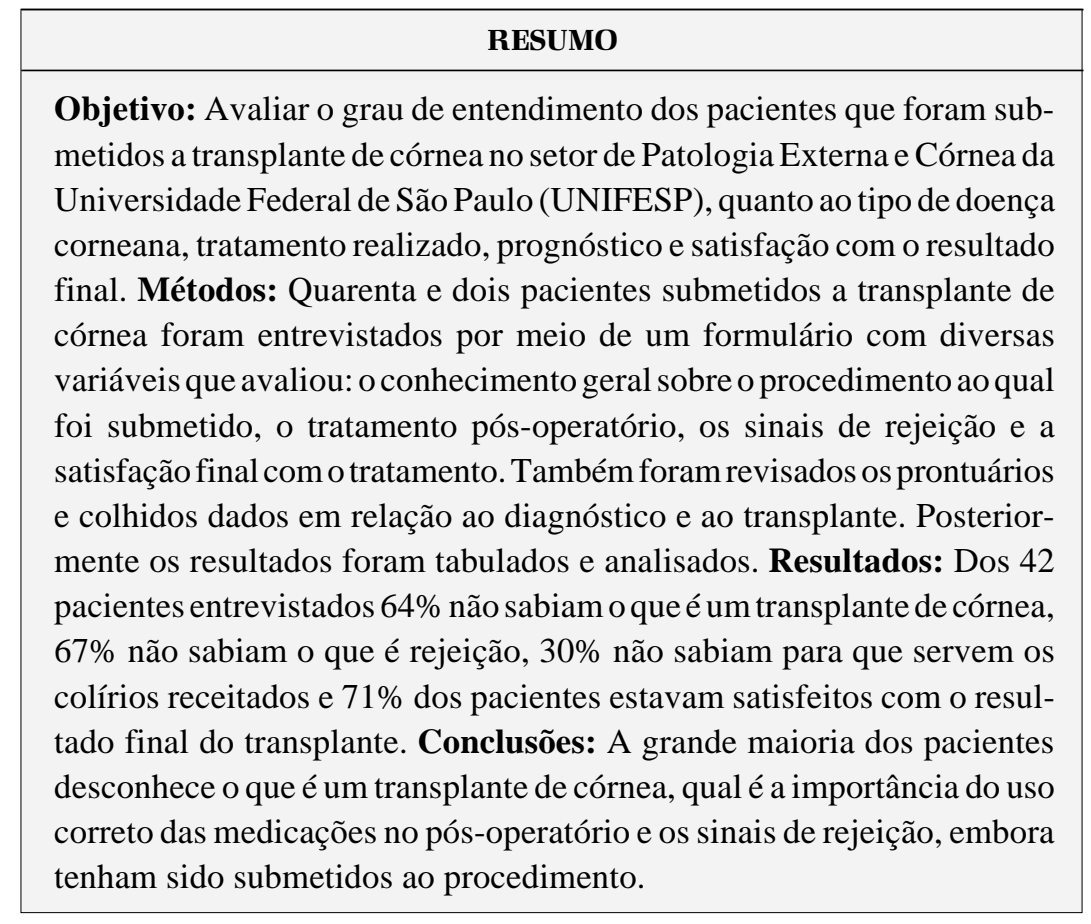

Descritores: Transplante de córnea; Soluções oftálmicas; Resultado de tratamento; Questionários; Satisfação do paciente; Rejeição de enxerto

\section{INTRODUÇÃO}

Trabalho realizado no Setor de Córnea e Patologia Externa do Departamento de Oftalmologia da Universidade Federal de São Paulo - UNIFESP.

${ }^{1}$ Fellow do ambulatório de Córnea e Patologia Externa da Universidade Federal de São Paulo - UNIFESP.

${ }^{2}$ Chefe do Setor de Córnea e Patologia Externa, Professora da Pós-graduação, Doutora em Oftalmologia pela Universidade Federal de São Paulo - UNIFESP.

${ }^{3}$ Livre Docente, Professora da Pós-graduação da Universidade Federal de São Paulo - UNIFESP.

${ }^{4}$ Doutor em Oftalmologia pela Universidade Federal de São Paulo - UNIFESP.

Endereço para correspondência: Gerson López Moreno Calle 46 norte No 8N-18 Urbanización El Bosque Cali - Colômbia - E-mail:gerson@oftalmo.epm.br; gerlopezm@hotmail.com

Recebido para análise em 24.09.2002

Versão revisada recebida em 14.04.2003

Aprovação em 09.05.2003

Nota Editorial: Pela análise deste trabalho e por sua anuência na divulgação desta nota, agradecemos ao Dr. Cláudio do Carmo Chaves.
O transplante de córnea é um dos procedimentos cirúrgicos mais importantes da oftalmologia pelo que representa na recuperação visual de um indivíduo na sociedade ${ }^{(1)}$. Em nosso dia-a-dia o transplante de córnea tem indicações claras com finalidades tectônicas e/ou óptica ${ }^{(2)}$. Todo paciente submetido a este tipo de cirurgia deve ter uma informação completa sobre o tipo de transplante ao qual será submetido, o cuidado pós-operatório e sinais de rejeição ${ }^{(3)}$, visto que o diagnóstico precoce pode alterar o resultado final do transplante, evitando que o paciente tenha novamente uma limitação visual com suas conseqüências sociais e alteração da qualidade de vida do individuo ${ }^{(4)}$.

O objetivo deste trabalho foi avaliar o grau de entendimento dos pacientes que foram transplantados no setor de patologia externa e córnea da Universidade Federal de São Paulo (UNIFESP), sobre o tipo de doença corneana, o tratamento realizado, prognóstico, e sua satisfação com o resultado final. 


\section{MÉTODOS}

Quarenta e dois pacientes submetidos ao transplante de córnea e que estão sendo acompanhados no ambulatório de Córnea e Patologia Externa da Universidade Federal de São Paulo (UNIFESP) foram entrevistados por meio de um formulário. O questionário foi aplicado por um único entrevistador. Foram analisadas as seguintes variáveis: sexo, idade, etnia, profissão, distância do local de atendimento em tempo, número de médicos pelos quais já foi atendido, de onde foi encaminhado, medicamento que atualmente está usando, se deixou de usar a medicação e porque, se sabe o que é um transplante, qual o tipo de doença e o tempo de evolução, há quanto tempo foi transplantado, número de transplantes realizados, motivos do primeiro e segundo transplantes, conhecimento sobre o tipo de transplante, sinais de rejeição, função dos colírios, astigmatismo, se está usando óculos, lente de contato ou nenhum e por último, satisfação com o resultado do tratamento. Posteriormente estas variáveis foram tabuladas e analisadas.

\section{RESULTADOS}

Foram operados 46 olhos de 42 pacientes, 26 eram de sexo masculino e 16 de sexo feminino (Tabela 1 ). A média de idade foi de 58,13 anos \pm 10 anos (variação de 2 a 80 anos) (Tabela 2).

Com relação à distribuição étnica, a cor parda apresentou a maior freqüência 51\%, seguida da branca com 40\%, preta $7 \%$ e amarela com $2 \%$.

Vinte (48\%) pacientes eram aposentados, 8 (19\%) pacientes trabalham em atividades do lar, 14 (33\%) exerciam outras atividades profissionais, sendo 4 estudantes (universitários; primeiro grau e segundo grau) (Gráfico 1).

A maioria dos pacientes (49\%) demora entre 1 a 2 horas para chegar ao ambulatório, 36\% chegam entre 2 a 5 horas,

\begin{tabular}{|c|c|}
\hline Sexo & Número de pacientes \\
\hline Masculino & $26 \quad(62 \%)$ \\
\hline Feminino & $16 \quad(38 \%)$ \\
\hline Total & $42(100 \%)$ \\
\hline
\end{tabular}

\begin{tabular}{|c|c|}
\hline Idade em anos & Número de pacientes \\
\hline 2 a 10 & $(2 \%)$ \\
\hline 11 a 20 & $(7 \%)$ \\
\hline 21 a 51 & $17 \quad(41 \%)$ \\
\hline 51 a 70 & $10 \quad(24 \%)$ \\
\hline 71 ou mais & $11 \quad(26 \%)$ \\
\hline Total & $42(100 \%)$ \\
\hline
\end{tabular}

$10 \%$ dos pacientes chegam em menos de uma hora e 5\% demoram mais de 6 horas (Gráfico 2).

Noventa e seis por cento dos pacientes foram atendidos por mais de três médicos, $2 \%$ por um único médico e $2 \%$ por dois médicos. Noventa por cento foram encaminhados por serviço público e somente $10 \%$ dos pacientes vinham encaminhados por um serviço privado (Gráfico 3).

No momento da entrevista $76 \%$ dos pacientes afirmaram estar usando medicação, dos quais $26 \%$ usavam colírio de corticóide como única medicação, 13\% estavam usando colírio que combinava antibiótico e corticóide, 19\% estavam usando colírio que combinava corticóide e lubrificante, 9\% estavam usando algum tipo de colírio não especifico para pós-operatório de transplante, $9 \%$ estavam usando corticóide tópico mais lubrificante, $3 \%$ estavam usando um antiglaucomatoso mais algum tipo de colírio que não correspondia ao tratamento específico para um paciente transplantado, 3\% usava corticóide sobre forma de colírio e oral mais um antiglaucomatoso, 9\% estavam usando unicamente colírio antiglaucomatoso, 9\% usavam antiglaucomatoso mais outro tipo de colírio não espe-

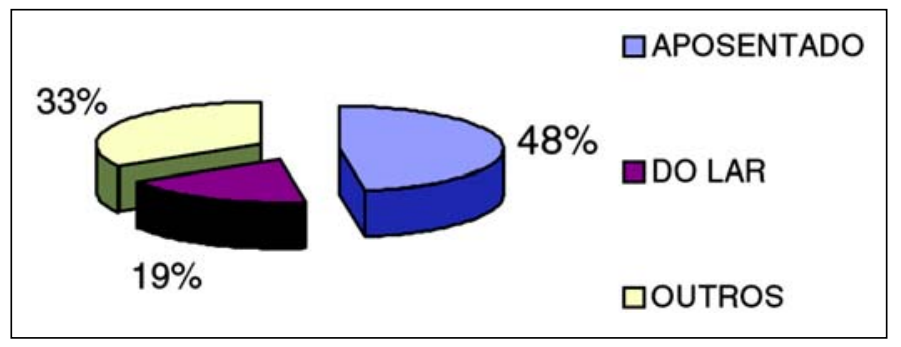

Gráfico 1 - Distribuição dos pacientes submetidos ao transplante de córnea quanto à profissão

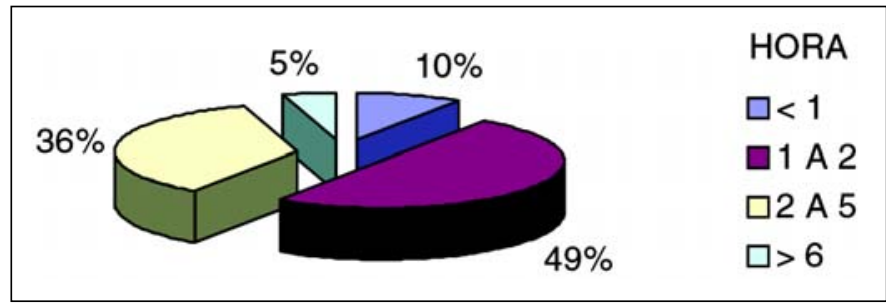

Gráfico 2 - Distribuição dos pacientes submetidos ao transplante de córnea quanto à distância, em horas, do domicilio até o ambulatório

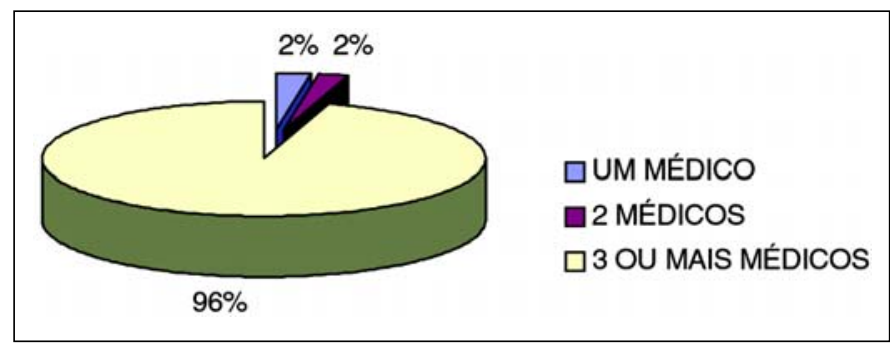

Gráfico 3 - Distribuição dos pacientes submetidos ao transplante de córnea em relação número de médicos examinadores 
cifico para pós-operatório de transplante (Gráfico 4). Vinte e quatro por cento suspenderam a medicação, dos quais $60 \%$ o fizeram por ordem médica, $20 \%$ por não notarem melhora de suas queixas, $10 \%$ por não saberem que tinham que continuar o uso do medicamento (Gráfico 5), e 10\% por falta de condição financeira para adquirir o medicamento.

Sessenta e quatro por cento dos pacientes não sabiam o que é um transplante de córnea, enquanto 36\% tinham algum conhecimento sobre o procedimento (Gráfico 6).

Quarenta e dois por cento dos pacientes relataram saber o diagnóstico da doença que os levou a fazer o transplante há onze anos ou mais, 29\% entre 2 a 5 anos, 24\% 6 a 10 anos, 5\% sabiam o diagnóstico há menos de um ano. Setenta e nove por cento foram transplantados há mais de um ano. Sessenta e quatro por cento dos pacientes submeteram-se ao transplante em um dos olhos, $17 \%$ em ambos os olhos, $19 \%$ foram transplantados duas ou mais vezes em um dos olhos (Gráfico 7). Vinte por cento dos pacientes realizaram transplante de córnea por apresentar ceratocone, $14 \%$ por úlcera de córnea, 14\% por algum tipo de distrofia, $10 \%$ por trauma químico, $10 \%$ por algum tipo de degeneração, 10\% por apresentar leucoma, 10\% falência primária, $7 \%$ por ceratopatia bolhosa e $5 \%$ por rejeição.

Noventa e cinco por cento dos pacientes não sabiam que tipo de transplante lhes foi realizado. Sessenta e sete por cento dos pacientes não sabiam o que é rejeição (Gráfico 8) e $36 \%$ afirmaram que já lhes havia sido explicado alguma vez os sinais de rejeição (Gráfico 9). Dos pacientes que relatavam conhecer os sinais de rejeição, 33\% lembravam de diminuição da acuidade visual e hiperemia como os principais sinais; $20 \%$ de diminuição da acuidade visual, dor ocular e hiperemia; 13\% de diminuição da acuidade visual; 13\% de diminuição da acuidade visual e dor ocular; 7\% de dor ocular e hiperemia; 7\% de hiperemia e 7\% não lembravam nenhum sinal (Gráfico 10).

Noventa e oito por cento dos pacientes operados não sabiam

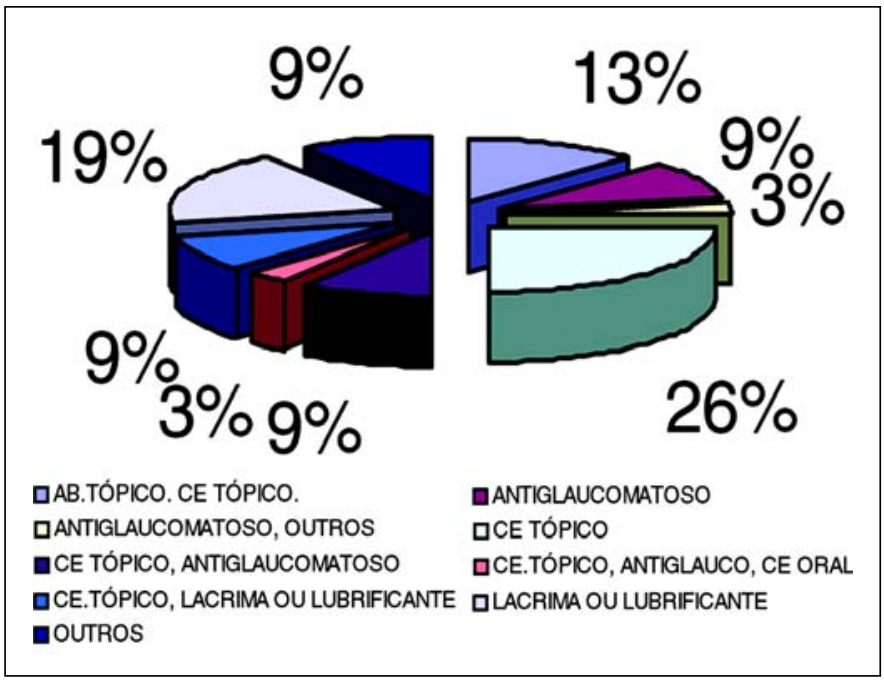

Gráfico 4 - Distribuição dos pacientes submetidos ao transplante de córnea em relação ao tipo de medicamento. ( $A B=$ Antibiótico; CE= Corticóide)

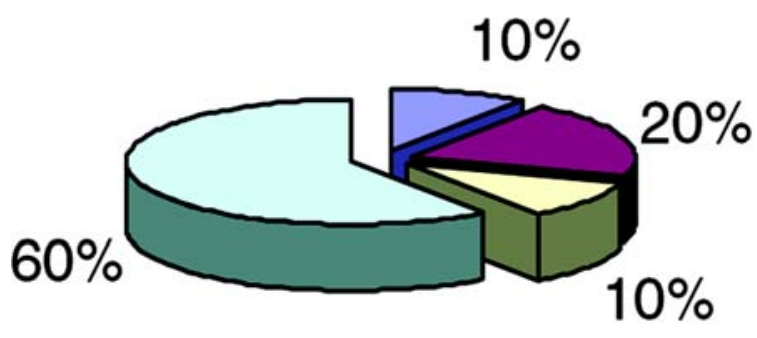

IFALTA DE DINHERO INĀO SABIA QUE TINHA QUE CONTINUAR 口NÃO NOTOU MELHORA ISUSPENSO PELO MÉDICO

Gráfico 5 - Distribuição dos pacientes submetidos ao transplante de córnea em relação aos motivos da não aderência utilização da medicação prescrita

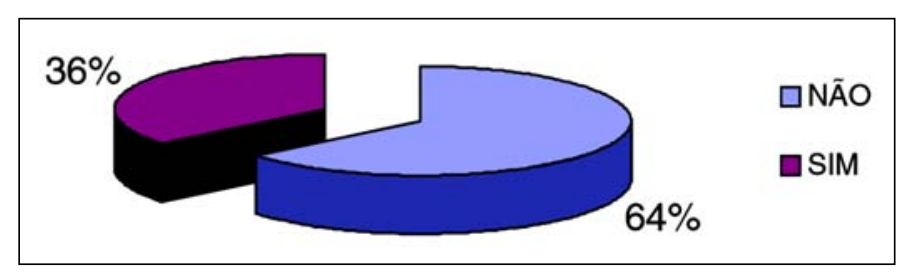

Gráfico 6 - Distribuição dos pacientes submetidos ao transplante de córnea em relação ao seu conhecimento sobre o procedimento

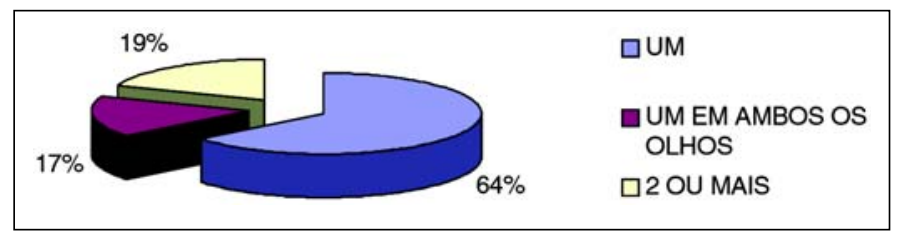

Gráfico 7 - Distribuição dos pacientes submetidos ao transplante de córnea em relação ao número de transplantes realizados

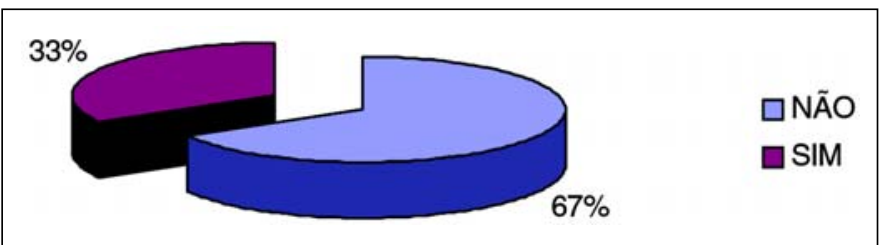

Gráfico 8 - Distribuição dos pacientes submetidos ao transplante de córnea quanto ao seu conhecimento sobre rejeição de transplante

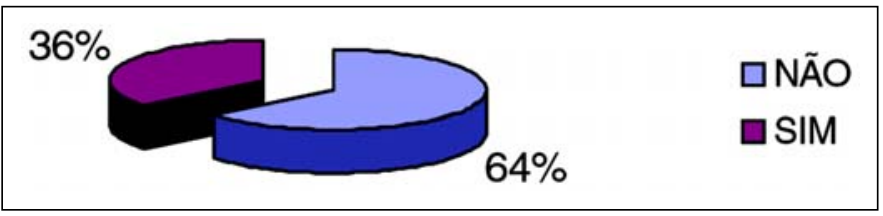

Gráfico 9 - Distribuição dos pacientes submetidos ao transplante de córnea em relação ao recebimento de explicação sobre os sintomas de rejeição 


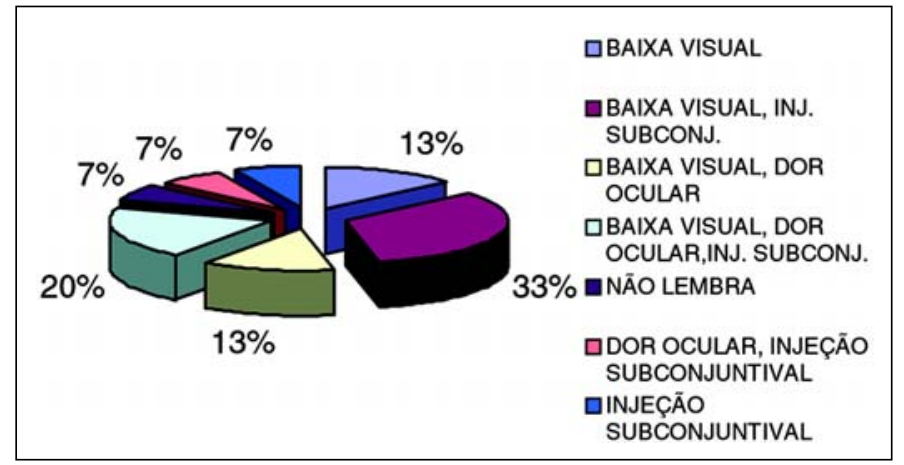

Gráfico 10 - Distribuição dos pacientes submetidos ao transplante de córnea em relação aos sintomas de rejeição que foram lembrados

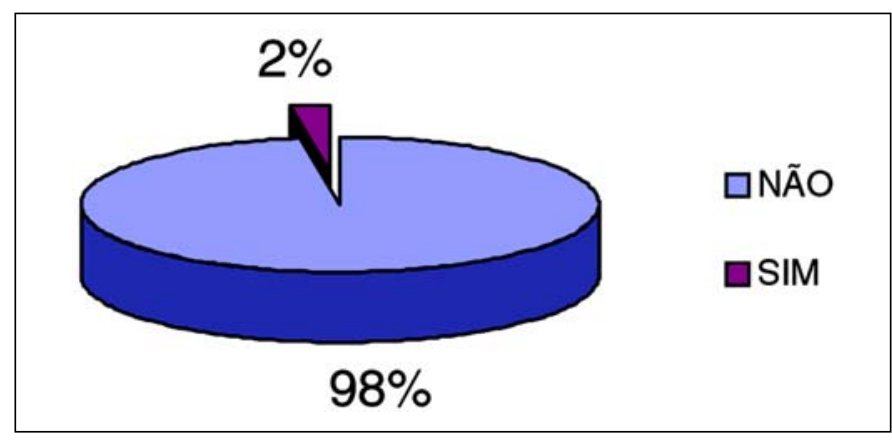

Gráfico 11 - Distribuição dos pacientes submetidos ao transplante de córnea quanto ao seu conhecimento sobre a durabilidade do transplante

qual seria a durabilidade de um transplante de córnea (Gráfico 11).

Noventa e oito por cento dos pacientes não sabiam o que é astigmatismo. Em relação à utilização de colírio trinta e um por cento não sabiam para que serviam os colírios, 19\% referiram outro tipo de função não correspondente à realidade, $17 \%$ imaginavam que seu uso era para melhorar a visão, 7\% para melhorar a visão e outras, 7\% para prevenir infecção e outras, 5\% para prevenir infecção, 5\% para prevenir infecção e rejeição, $5 \%$ para prevenir rejeição, 2\% para prevenir rejeição e outros, 2\% para melhorar a visão e prevenir rejeição (Gráfico 12).

Sessenta e quatro por cento dos pacientes não estavam usando nenhum tipo de correção óptica, 24\% estavam usando óculos, $10 \%$ lente de contato e $2 \%$ dos pacientes estavam usando óculos e lentes de contato (Gráfico 13). Setenta e um por cento dos pacientes estavam satisfeitos com o resultado final do transplante e, $29 \%$ não estavam satisfeitos com o resultado do procedimento (Gráfico 14).

\section{DISCUSSÃO}

Segundo a literatura a indicação de transplante tem aumentado muito nos últimos 40 anos e é uma das cirurgias mais realizadas $^{(5)}$ e isto se deve ao aumento da faixa etária de nossos pacientes e aos novos critérios de inclusão para a realização de transplante ${ }^{(6)}$. A maioria dos pacientes era do sexo

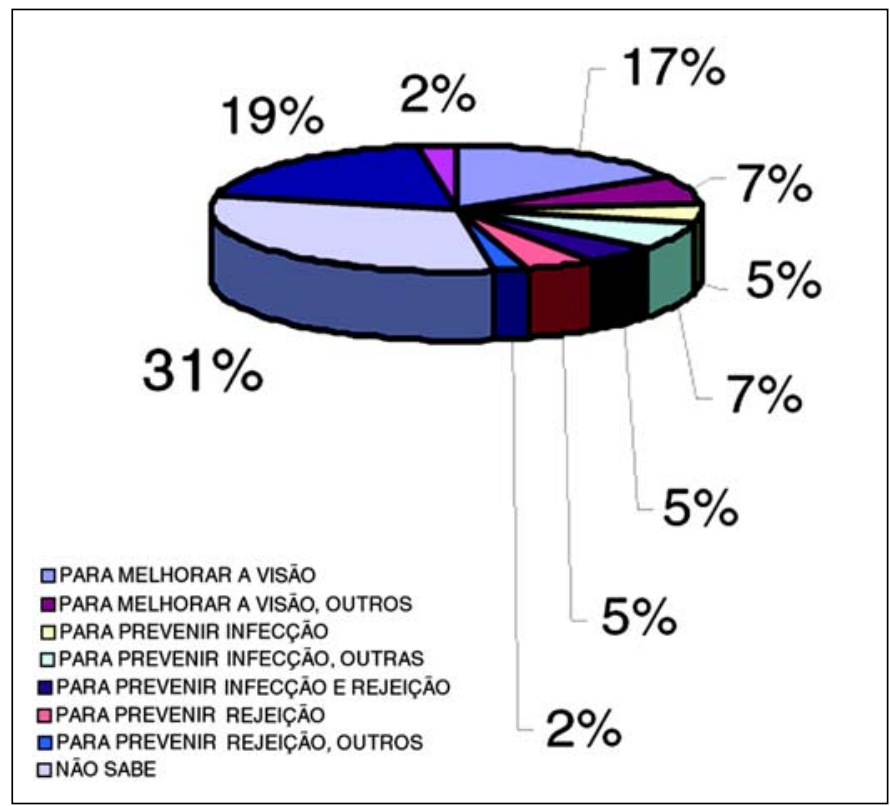

Gráfico 12 - Distribuição dos pacientes submetidos ao transplante de córnea quanto ao seu conhecimento sobre a função dos colírios

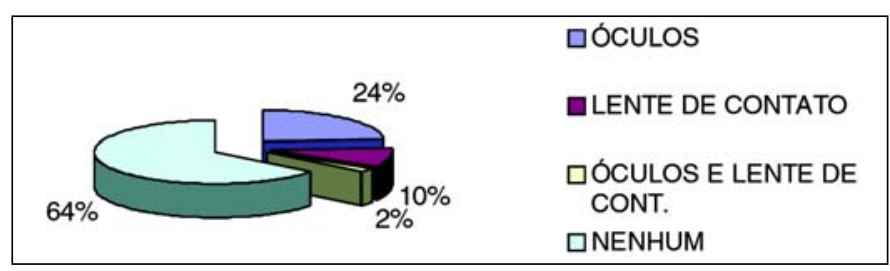

Gráfico 13 - Distribuição dos pacientes submetidos ao transplante de córnea quanto ao tipo de correção óptica em uso após o transplante

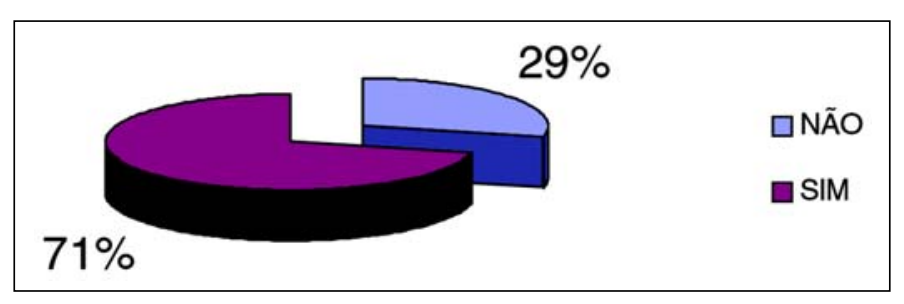

Gráfico 14 - Distribuição dos pacientes submetidos ao transplante de córnea quanto à satisfação em relação ao resultado visual final após o transplante

masculino, com média de idade de 58,13 e, embora grande parte destes fossem aposentados, ainda apresentavam potencial de produtividade socioeconômica. Quarenta e nove por cento dos pacientes demoram entre 1 e 2 horas para chegar ao ambulatório, o que facilita o acompanhamento destes pacientes. Noventa e seis por cento dos pacientes foram atendidos por mais de três médicos durante o acompanhamento devido ao modo de funcionamento do nosso sistema ambulatorial. Já que é importante o aprendizado do paciente a cada consulta consecutiva sobre o procedimento ao qual foi submetido e a confiança que é adquirida pelo paciente, à medida que segue 0 
seu tratamento, sendo orientado e atendido pelo mesmo médico. Setenta e seis por cento dos pacientes estavam usando algum tipo de colírio os quais, em sua maioria eram corticóide mais lubrificante ocular, por serem medicamentos usados rotineiramente no pós-operatório imediato e tardio do transplante de córnea. Outro dado que chamou muita atenção é que 31 \% dos pacientes não tinham uma idéia clara da função dos colírios, fato de fundamental importância para a recuperação, já que esta depende da correta aplicação dos colírios, e se os pacientes estivessem bem informados sobre a função dos colírios estariam mais cientes da sua importância e poderiam aplicá-los de maneira correta. Vinte e quatro por cento dos pacientes suspenderam a medicação por diversos motivos, destes $10 \%$ o fizeram por que não notaram melhora ou não sabiam que tinham que continuar, sua utilização, o que é reflexo da desinformação dos pacientes, prejudicando a adequada recuperação dos mesmos. Em um trabalho realizado por Paulino et al concluíram que pacientes glaucomatosos atendidos em Hospital Universitário Público não têm recebido orientação satisfatória quanto à dosagem e técnica de instilação de colírios, sendo importante prestar uma maior atenção ao tema "aderência” resultando como benefício importante na progressão da lesão glaucomatosa ${ }^{(7)}$. A mesma conclusão também é aplicável para os pacientes que são submetidos ao transplante de córnea.

Sessenta e quatro por cento dos pacientes não sabiam o que é um transplante embora tenham se submetidos a um procedimento importante, novamente demonstrando a deficiência na comunicação entre o médico e o paciente ${ }^{(8)}$. Sessenta e sete por cento dos pacientes não sabiam o que é rejeição e o restante lembrava apenas de alguns sinais. Noventa e oito por cento dos pacientes não sabiam o que é astigmatismo, embora seja um dado importante, pois influencia na qualidade de visão final.

Quarenta e dois por cento dos pacientes tinham onze ou mais anos de diagnóstico da doença de base que os obrigou a realizar o transplante, e isso talvez se deva à longa espera que os pacientes enfrentam para se submeterem ao procedimento.

Sessenta e quatro por cento dos pacientes não estavam usando nenhum tipo de correção óptica, talvez devido à dificuldade existente na realização de refração ou adaptação com lente de contato. Apesar do que descrevemos acima, 71\% dos pacientes estavam satisfeitos com o resultado final do procedimento. Em estudo realizado por Lipener et al, foi observado $46 \%$ no sucesso de adaptação de lentes de contato em pacientes transplantados, promovendo acuidade visual igual, ou melhor, que 20/40 em 55\% dos casos, o que demonstra a importância de uma boa avaliação refracional destes pacientes quanto à qualidade visual final ${ }^{(9)}$.

\section{CONCLUSÃO}

Observamos que a grande maioria dos pacientes não tem entendimento suficiente sobre o tipo de doença corneana, procedimento cirúrgico ao qual foi submetido e os cuidados necessários no pós-operatório embora a maioria está satisfeita com o resultado final mesmo sem correção óptica adequada. Definitivamente, os pacientes precisam de uma melhor informação prévia ao tratamento cirúrgico por parte da equipe médica. Os pacientes que estão aguardando o procedimento deverão receber palestras educativas por médicos e pessoais treinados de enfermagem e tecnólogos. A comunicação médico-paciente deve ser melhorada, e o sistema de atendimento ambulatorial deve ser mudado visando uma melhora na qualidade de atendimento do paciente.

\section{ABSTRACT}

Purpose: To evaluate the understanding of the patient submitted to corneal transplant regarding the type of surgery, used treatment, rejection signs and final outcome satisfaction, at Cornea and External Disease Sector of the Federal University of São Paulo (UNIFESP). Methods: 42 patients submitted to corneal transplant were interviewed using a questionnaire that includes multiple variables regarding knowledge on the general procedure, postoperative treatment, rejection signs and satisfaction with the final outcome. We also collected data about the diagnosis and the transplant. The data were tabulated and analyzed. Results: $67 \%$ of 42 interviewed patients did not know what a corneal transplant is, $67 \%$ did not know about rejection signs, 30\% did not know the purpose of postoperative medicines and $71 \%$ were satisfied with the final outcome. Conclusions: Most of transplanted patients do not know the meaning of corneal transplant, rejections signs and the correct use of medicines but are satisfied with the final outcome.

Keywords: Corneal transplantation; Ophthalmic solutions; Treatment outcome; Questionnaires; Patient satisfaction; Graft rejection

\section{REFERÊNCIAS}

1. Arentsen JJ, Laibson PR. Surgical management of pseudophakic corneal edema: Complications and visual results following penetrating keratoplasty. Ophthalmic Surg 1982;13:371-3.

2. Nosé W, Nosé RA. Ceratoplastias e ceratectomias. In: Belfort Jr R, Kara José-N. Córnea clínica - cirúrgica. São Paulo: Roca; 1996. p.493-504.

3. Lima CA, Ferras EVAP, Sobrinho M, Kara JN. O quê os portadores de ceratocone sabem sobre sua doença? [resumo]. Arq Bras Oftalmol 1999;62:419.

4. Corpa V, Lima NV, Schellini SA. Doação e transplante de córnea: visão do paciente [resumo]. Arq Bras Oftalmol 1999;62:374.

5. Acedo JT. Queratoplastias y queratoprotesis. Barcelona: Edikamed; 1992.

6. Lindquist TD, Mcglothan JS, Rotkis WM, Chandler JW. Indications for penetrating keratoplasty: 1980-1988. Cornea 1991;10:210-6.

7. Paulino V, Vasconcelos JPC, Pelegrino M, Kara-Jose N. Análise do método de aplicação do colírio em pacientes glaucomatoso [resumo]. Arq Bras Oftalmol 1994;57:123.

8. Conselho Regional de Medicina do Estado de São Paulo. O que melhora a relação médico-paciente, por parte do médico. Guia da Relação médico-paciente. São Paulo; 2001. p.7.

9. Lipener C, Kwitko S, Uras R, Zamboni F, Levinski R, Araújo AF, Lima Jr RP. Adaptação de lentes de contato em pacientes pós-transplante de córnea. Arq Bras Oftalmol 1990;53:41-4. 